\title{
The influence of the spatial frequency content of discrete roughness distributions on the development of the crossflow instability
}

\author{
Evelien van Bokhorst ${ }^{1}$, Marco Placidi ${ }^{2}$ and Chris Atkin ${ }^{3}$ \\ School of Mathematics,Computer Science and Engineering \\ City University London, Northampton Square, EC1V OHB, London
}

\begin{abstract}
An experimental investigation on the influence of the spatial frequency content of roughness distributions on the development of crossflow instabilities has been carried out. From previous research it is known that micro roughness elements can have a large influence on the crossflow development. When the spanwise spacing is chosen such that it is the most unstable wavelength (following linear stability analysis), stationary crossflow waves are amplified. While in earlier studies the focus was on the height or spanwise spacing of roughness elements, in the present study it is chosen to vary the shape of the elements. Through the modification of the shape the forcing at the critical wavelength is increased, while the forcing at the harmonics of the critical wavelength is damped. Experiments were carried in the low turbulence wind tunnel at City University London $(T u=0.006 \%)$ on a swept flat plate in combination with displacement bodies to create a sufficiently strong favourable pressure gradient. Hot wire measurements across the plate tracked the development of stationary and travelling crossflow waves. Initially, stronger crossflow waves were found for the elements with stronger forcing, while further downstream the effect of forcing diminished. Spatial frequency spectra showed that the stronger forcing at the critical wavelength (via the roughness shape) dominates the response of the flow while low forcing at the harmonics has no notable effect. Additionally, high resolution streamwise hot wire scans showed that the onset of secondary instability is not significantly influenced by the spatial frequency content of the roughness distribution.
\end{abstract}

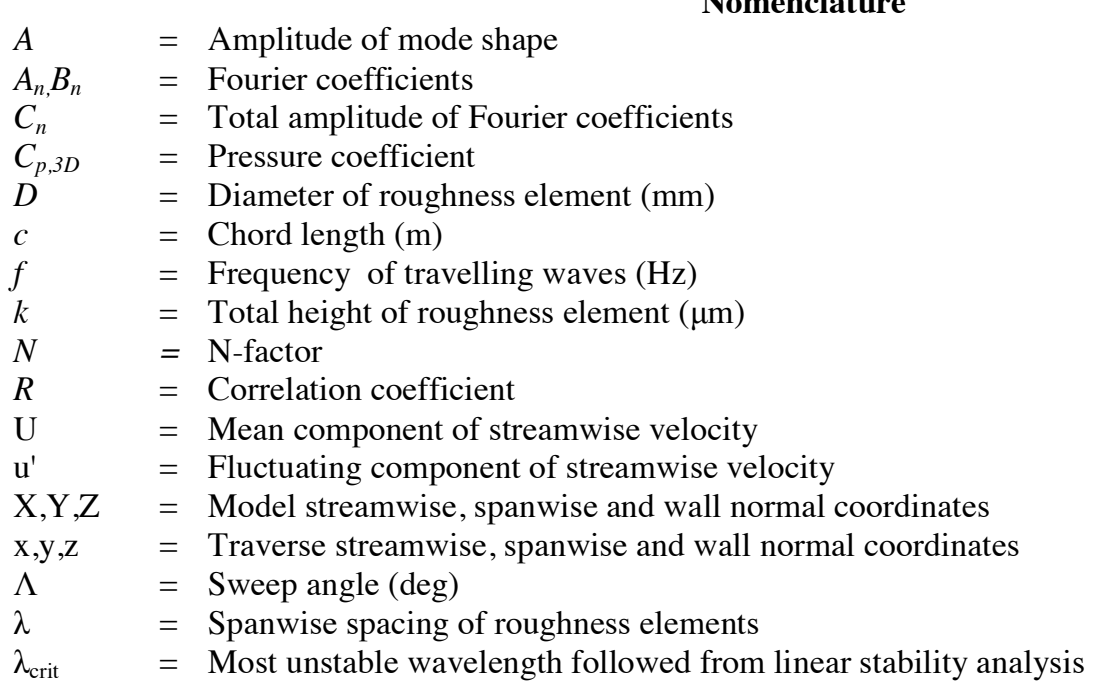

\footnotetext{
${ }^{1}$ Ph.D. candidate, SMCSE Northampton Square, EC1V 0HB.

${ }^{2}$ Research Fellow, SMCSE Northampton Square, EC1V 0HB.

${ }^{3}$ Professor of Aeronautical Engineering., SMCSE Northampton Square, EC1V 0HB. 1 


\section{Introduction}

$\mathrm{T}$ RANSITION from laminar to turbulent flow can follow several paths depending on the level of environmental disturbances ${ }^{1}$. During flight, these disturbances are expected to be sufficiently small, such that natural transition to turbulence occurs. The first stage of this natural transition process to turbulence is the "receptivity" process" Here, instability waves are generated from external disturbances, such as, freestream turbulence or surface roughness. If the disturbances are able to feed into the boundary layer and act upon its unstable eigenmodes, the boundary layer is said to be receptive to external inputs. The second stage of this transition process is the growth of the primary instability: firstly in a linear and secondly in a non-linear manner. Depending on the geometry and the pressure gradient, characterizing the problem, different types of instabilities can be amplified. Finally, after these stages, a high-frequency secondary mechanism appears and the flow breaks down into turbulence. While the possible transition paths are known, it is still unclear how external disturbances exactly influence the receptivity process and ultimately the transition location ${ }^{3}$. Understanding the latter is of interest to aeroplane manufacturers, as extensive laminar flow can lead to significant reduction in fuel consumption and operating costs, due to the lower skin friction $\mathrm{drag}^{4}$.

The focus of the current study is on the influence of discrete surface roughness distributions on the development of the crossflow instabilities. Crossflow instabilities dominate the transition process over swept wings and therefore most accurately represent the flow over an aeroplane wing.

The crossflow instability is an inviscid-type instability, which occurs due to a combination of sweep angle and favourable pressure gradient, as was first identified by Gray ${ }^{5}$. Figure 1 shows a schematic of the origin of the crossflow instability. At the attachment line, only a velocity component parallel to the leading edge is present. Then, the flow is accelerated in the streamwise direction, causing the inviscid streamlines to curve. Due to viscous effects, this streamlines curvature induces a flow component perpendicular to the streamline inside the boundary layer. This crossflow velocity, $u_{C F}$, is zero both at the wall (due to the no slip condition) and at the edge of the boundary layer, where the pressure gradient is balanced by the centripetal acceleration. This results in an inflection point in the velocity profile, which is a source for an inviscid instability (following Rayleigh's criterion ${ }^{6}$ ). The instability manifests itself as steady and unsteady co-rotating vortices, almost aligned to the potential flow direction. These vortices were firstly visualised by Gregory et al. ${ }^{7}$ and Poll ${ }^{8}$. The amplitude of the vortices first grows linear and later in a non-linear manner. When the stationary instability distorts the mean flow, inflection points will emerge in the mean velocity profiles. These inflection points are the source of a secondary instability, which manifests itself as a very high-frequency travelling wave (as first identified by Kohama ${ }^{9}$ ). Two types of secondary instabilities are recognized. Type I, with frequency $f$, is found in regions with high shear caused by $D U / D Z$ gradients and Type II, with a frequency of approximately $2 f$, is characteristic of regions with high shear caused by $D U / D Y$ gradients ${ }^{10}$. The secondary instability grows rapidly and a few percent downstream the flow breaks down ${ }^{11}$.

It should be noted that in addition to the crossflow instability, Tollmien-Schlichting (T-S) waves might appear over a swept or curved body ${ }^{12,13}$. This is a viscous-type of instability, which is promoted in adverse pressure gradient flows. In the current experimental design the growth of the T-S waves should therefore be managed to ensure that a crossflow dominated flow is created. From numerical linear stability analysis, it follows that the most amplified crossflow disturbance is the unsteady travelling wave, which should therefore dominate transition ${ }^{14}$. However, from experiments and computational studies it was found that this strongly depends on the receptivity process and particularly on the level of freestream turbulence intensity. For a low-turbulence environment $(\mathrm{Tu}<0.1-0.2 \%)$ the flow is dominated by the stationary waves, whilst for higher turbulence levels, the travelling crossflow becomes dominant $^{15,16,17,18,19,20}$. In flight conditions, the turbulence levels are expected to be $10 w^{21}$ and therefore the stationary crossflow wave should dominate the transition process. 


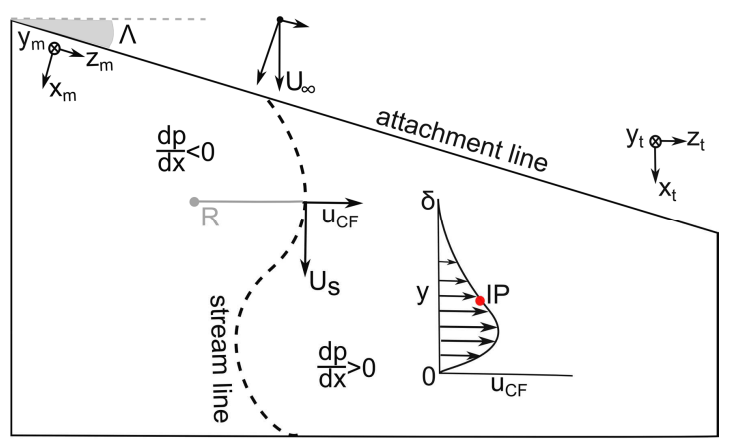

Figure 1. Schematic representation of the origin of the crossflow instability.

In roughness receptivity studies simple surface roughness distributions are usually investigated. In these studies, discrete roughness elements $(D=O(\mathrm{~mm}), k=O(\mu \mathrm{m})$, where $D$ is the diameter of the roughness and $k$ the height) are placed close to the leading edge (near to the neutral stability point) at a certain spanwise spacing. Numerically, it has been found that, under the parallel-flow assumption, the response of the boundary layer on the small roughness elements can be computed using Fourier transform theory ${ }^{18,22,23}$. The response of the flow is dominated by the leaststable eigenmode having the same spanwise wavenumber, $\beta$, and frequency, $\omega$, as the roughness distribution ${ }^{23}$. Experimentally, this has first been shown by Reibert et al. ${ }^{24,25}$, who found that roughness elements spaced at spanwise spacing $\lambda$ only excite the fundamental and sub-harmonic disturbances with wavelengths $\lambda \vee n$, whilst the disturbance with wavelengths greater than $\lambda$ are not amplified. These conclusions have some interesting implications. Firstly, they greatly simplify the experimental data acquisition, since the flow is forced to be spanwise uniform, just a few wavelengths need to be investigated. Secondly, if $\lambda$ is chosen such that it does not amplify the most unstable eigenmode, transition can be controlled and even delayed ${ }^{26,27,28}$. Other experimental studies showed that the ratio of diameter $\mathrm{D}$ and spanwise spacing $\lambda$ of a single element has a strong influence on the transition location $^{29,30}$. When a two-dimensional roughness strip is used there is no influence of the transition location ${ }^{30}$. Both these observations can again be attributed to the coupling between the spectrum of the roughness element and the underlying flow. It also indicates that the spatial frequency content of the roughness distribution has a stronger influence on the transition process than the frontal area or volume of the roughness. Other discrete roughness elements studies have shown that the elements are most effective when placed closely to the neutral stability point $^{17,30}$

Furthermore, increasing the height of the roughness elements increases the initial amplitude of the primary stationary crossflow instability ${ }^{18,30,31}$. However, if non-linear amplitude saturation of the stationary crossflow waves occurs the roughness height does not influence the transition location ${ }^{24}$. White and Saric ${ }^{11}$ found that increasing the roughness height lead to increased energy in the low-frequency domain. Furthermore, when the roughness height was increased, the most dominant secondary instability mode shifted from type I to type II.

Real surface roughness found on airliners, has a highly random nature and is therefore more complex to investigate. It is found experimentally that a small increase in roughness height $(O(\mu \mathrm{m}))$ significantly increases the amplitude of the stationary vortex and contributes to move the transition location forward. Hence, the boundary layer becomes more receptive ${ }^{17,30}$. Mughal and Ashworth ${ }^{32}$ studied random roughness distributions through a stochastic technique. They found that if the roughness distribution had a larger Root Mean Square (RMS) height, this did not always have a stronger effect on the flow. They concluded that the forcing through the spectral content of the roughness distribution is more important.

Most of the previous experimental roughness studies have been carried out based on discrete roughness distributions. In these studies mostly the height of the roughness elements and spanwise spacing of elements is studied. The use of discrete roughness elements simplifies the experimental procedure, thus omitting parts of the physics occurring on an actual aeroplane wings. Furthermore, most experiments are carried out in the linear growth phase of the crossflow instability waves, however there is less focus on the non-linear growth phase and break down into turbulence.

In contrast, the current experimental work studies the effect of modulating the spatial frequency content of the roughness distribution by changing the shape of the roughness element. It is important to understand the effect of the spatial frequency content of the roughness elements since it might have a greater effect on the flow than other parameters such as height. From previous studies it is found that when the stationary crossflow wave starts to grow nonlinear, there are interactions of the $\lambda n$ modes $^{10,24}$. As shown in section IIB cylindrical roughness elements forces $\lambda / n$ modes strongly, which might be the reason they are coupled with the modes in the flow. To test this hypothesis 3

American Institute of Aeronautics and Astronautics 
pyramidal roughness elements are tested which have strong forcing at $\lambda$ but almost no forcing at $\lambda n$. Thus, the focus of this investigation is on the effect of the two roughness distributions on the growth and saturation of the stationary and travelling crossflow instability.

\section{Experimental apparatus and procedure}

\section{A. Experimental model}

It is clear from the literature that there are several setups to achieve a well-developed flow dominated by crossflow instabilities ${ }^{31,33}$. In this project it was chosen to use a displacement body (DB) placed above a swept flat plate, similar to the setup described in Nitschke-Kowsky ${ }^{33}$. The displacement body (typically a wing or a cylinder) creates a favourable pressure gradient $(d p / d x<0)$ on the swept flat plate, such that crossflow growth can be achieved. This setup was chosen in favour of a 3D curved aerofoil due of the reduced complexity in the traverse movements, as well as the possibility of changing the pressure gradient on the plate.

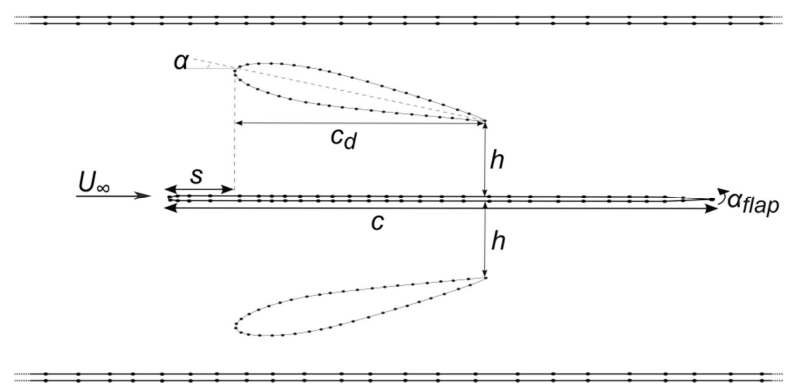

Figure 2: Panel code layout with the relevant parameters defined.

To design the displacement body a panel code was developed (Fig. 2). In the panel code the shape of the displacement body, the size of the displacement body, chord length $c_{d}$, the angle of attack, $\alpha$, the flap angle, $\alpha_{\text {flap }}$, the height above the plate, $h$, were varied and, for each case, the pressure distribution on the plate was calculated. The resulting pressure distribution was then compared to distributions found in literature ${ }^{30,33}$. Due to the inviscid nature of the panel code the pressure distribution is only an approximation of the actual distribution on the plate, since flow separation and boundary layer growth are not taken into account.

When a satisfactory pressure distribution was found, a Linear Stability Analysis (LSA) is performed to calculate the growth of Tollmien-Schlichting and crossflow waves. This growth is expressed with N-factors which is defined as the growth rates integrated over the streamwise distance, as first proposed by van Ingen and later implemented by Smith and Gamberoni ${ }^{34,35}$. The LSA consists of two stages. In the first stage the base flow is calculated with $B L 2 D^{36}$, which solves the compressible laminar boundary layer equations for swept-tapered or infinite swept wing. In the second stage results from $B L 2 D$ are fed into $C o D S^{37}$ to perform a linear stability analysis on threedimensional swept wings resulting in $\mathrm{N}$-factor curves for the Tollmien-Schlichting and crossflow instability waves. Following this analysis a $l w k 80100$ airfoil was chosen as displacement body with $c_{d}=0.2 c, \alpha=9^{\circ}$ and $h=0.1 \mathrm{~m}$.

A fixed swept flat plate $\left(\Lambda=45^{\circ}, c=1.6 \mathrm{~m}\right)$ was designed to be used in the experiments. This is in line with previous experimental work and the flow demonstrates industrially-relevant characteristics. An asymmetric leading edge (LE) has been designed (following Hanson ${ }^{38}$ ) to reduce the importance of the pressure gradient history, thus facilitating comparison between experimental and numerical results. This is also chosen to have minimal receptivity effects.

To assure high-surface quality the plate was progressively polished with progressively finer emery paper grits (from 400 to 1200). Small scratches were filled with car body filler (Simoniz spray putty). The discontinuity between the leading edge and plate was also removed following similar procedures. 


\section{B. Roughness distributions}

Two types of roughness distributions with different spatial frequency content are tested herein. In the first set of experiments, cylindrical roughness elements are taken into account since they have been investigated extensively in previous studies ${ }^{24,26,31}$. For these roughness elements, the height $(k)$, diameter $(D)$ and spanwise spacing $(\lambda)$ are the design parameters (Fig. 3). From linear stability analysis the most unstable wavelength $\left(\lambda_{\text {crit }}\right)$ is found to be around $11.5 \mathrm{~mm}$, which is chosen to be the spanwise spacing $\lambda$. Radeztsky ${ }^{30}$ found that the effect of roughness is significant for $D / \lambda=0.25-0.4$; which leads to a chosen diameter of $3 \mathrm{~mm}$. The height of one element, $k_{l}$, is chosen to be $6 \mu \mathrm{m}$, which is the smallest manufacturable size and is below the threshold for roughness causing bypass transition ${ }^{1}$. Two different heights are tested, where four will be stacked on top of each other $(k=24 \mu \mathrm{m})$ to create greater disturbances. The roughness elements, manufactured by Redd Europe Ltd, were printed on an A4-size transfer paper and applied in proximity of the neutral stability point, parallel to the leading edge of the plate.

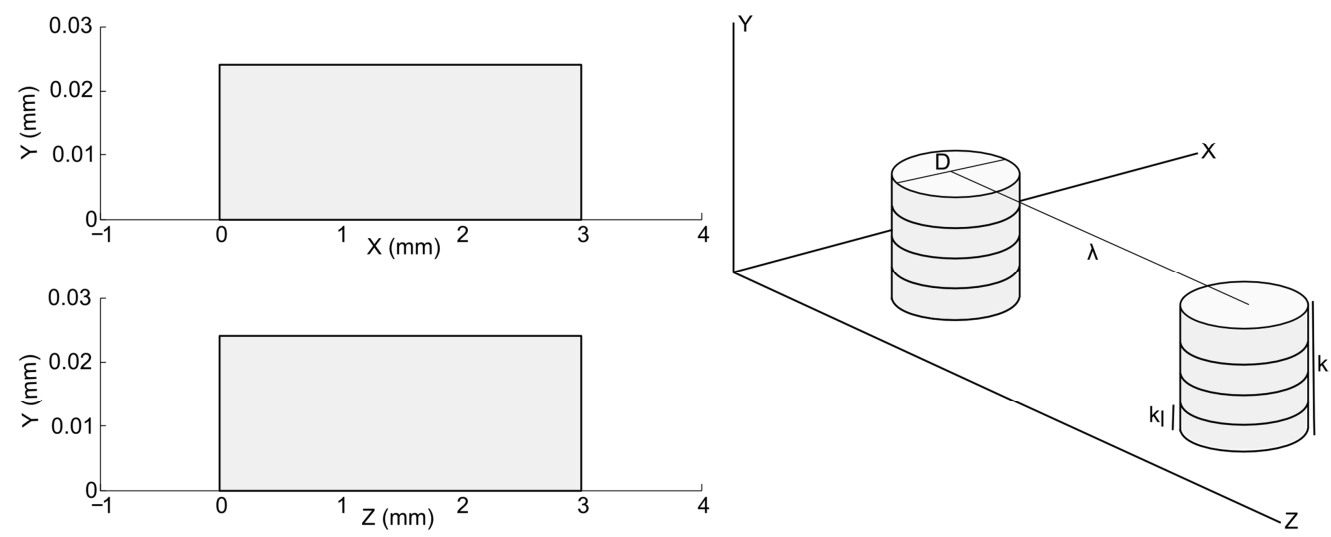

Figure 3. Cylindrical roughness distribution layout.

In the second set of experiments, more complex roughness distributions were designed. From computational studies and Fourier theory analysis, it follows that the spectrum of the roughness distribution is strongly coupled to the response of the boundary layer ${ }^{18,22,23}$. Experimentally this has been verified with discrete roughness elements by Radeztsky et al. ${ }^{30}$ (who varied the diameter of the discrete roughness elements) and by Reibert et al. ${ }^{24}$ (who varied the spanwise spacing of the roughness elements). However, more complex distributions with stronger forcing of the most unstable wavelength have not been tested. In this experiment it was chosen to create a discretized 3D roughness element, which is sinusoidal in the spanwise direction and has a smoothened step in the streamwise direction. Preferably, the roughness distributions would be machined, however this is a costly and lengthy procedure. Therefore it is chosen to discretize a sine wave, such that, it can be constructed of different layers of discrete roughness elements. The discretization resulted in a roughness element with $k=24 \mu \mathrm{m}$ and $\lambda=11.5 \mathrm{~mm}$. (Fig. 4). 

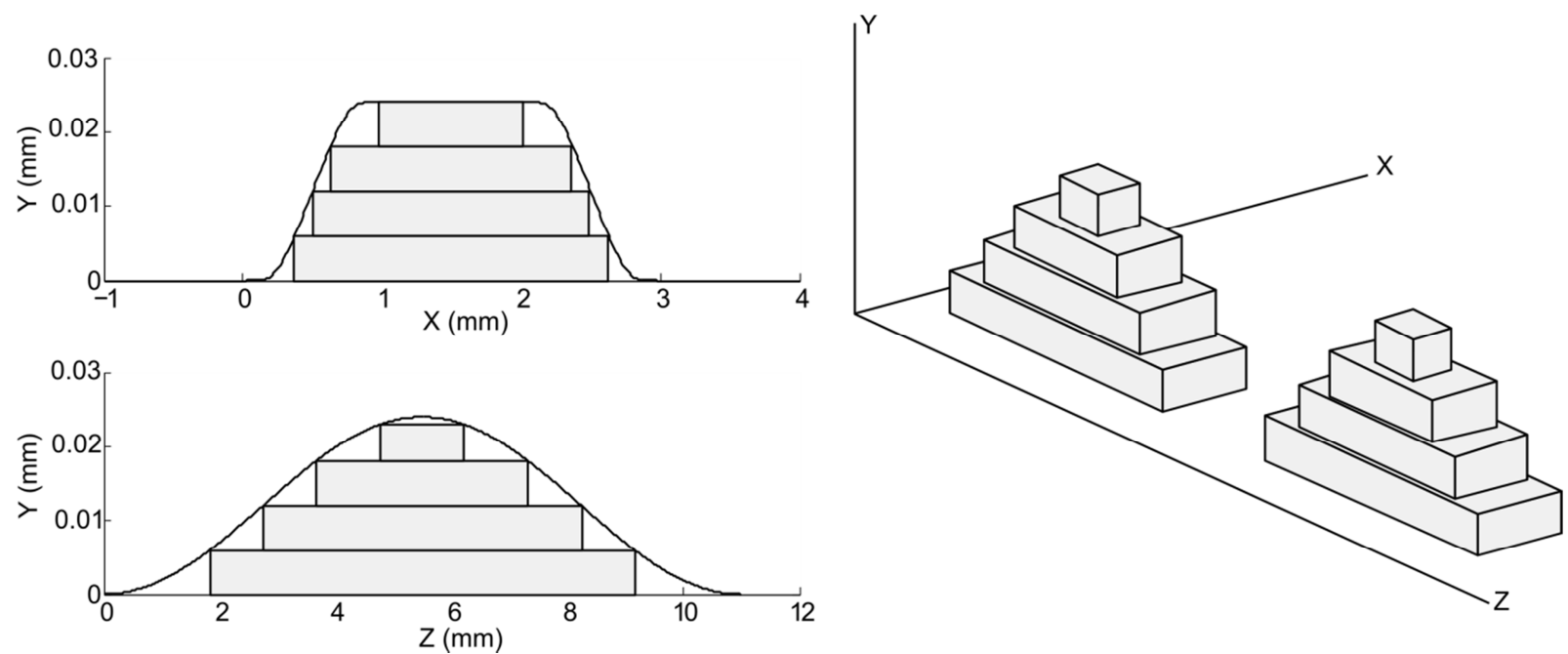

Figure 4: Pyramidal roughness distribution lay-out.

The amplitude spectrum of the roughness distributions was computed using standard Fourier series. For a circular, square or rectangular element with height $k$, diameter $D$ and spanwise spacing $\lambda$ the Fourier coefficients $A_{n}$ and $B_{n}$ are written as:

$$
\begin{aligned}
& A_{n}=\frac{2}{\lambda} \int_{0}^{\lambda} h(z) \cos \left(\frac{2 n \pi z}{\lambda}\right) d z=\frac{k}{n \pi} \sin \left(\frac{2 n \pi D}{\lambda}\right) \\
& B_{n}=\frac{2}{\lambda} \int_{0}^{\lambda} h(z) \sin \left(\frac{2 n \pi z}{\lambda}\right) d z=\frac{k}{n \pi}\left(1-\cos \left(\frac{2 n \pi D}{\lambda}\right)\right) .
\end{aligned}
$$

For $i$ elements stacked on top of each other, with each their own $D_{i}$ and $k_{i}$ the coefficients are added for each element. For example the amplitude of the pyramidal roughness distribution $A_{n, \Delta}$ results in:

$$
A_{n, \Delta}=\frac{k_{1}}{n \pi} \sin \left(\frac{2 n \pi D_{1}}{\lambda}\right)+\frac{k_{2}}{n \pi} \sin \left(\frac{2 n \pi D_{2}}{\lambda}\right)+\frac{k_{3}}{n \pi} \sin \left(\frac{2 n \pi D_{3}}{\lambda}\right)+\frac{k_{4}}{n \pi} \sin \left(\frac{2 n \pi D_{4}}{\lambda}\right) .
$$

The amplitudes $C_{n}$ are then computed with

$$
C_{n}=\sqrt{A_{n}^{2}+B_{n}^{2}},
$$

where the forcing is determined at wavelengths $\lambda / n$. In Figure 5 the amplitude spectrum is shown. As expected the amplitude for the pyramidal elements is higher at $\lambda(\mathrm{n}=1)$ than for the cylindrical elements. Furthermore, the $\lambda / n$ modes are damped for the pyramidal elements. The amplitude spectrum for roughness elements, which have a spanwise spacing is of $3 \lambda$ is also shown. This is similar to experiments carried out by Reibert et al. ${ }^{24}$. If the spanwise spacing is increased, additional modes are forced while for the pyramidal elements only $\lambda$ is stronger forced and $\lambda / n$ modes are damped. 


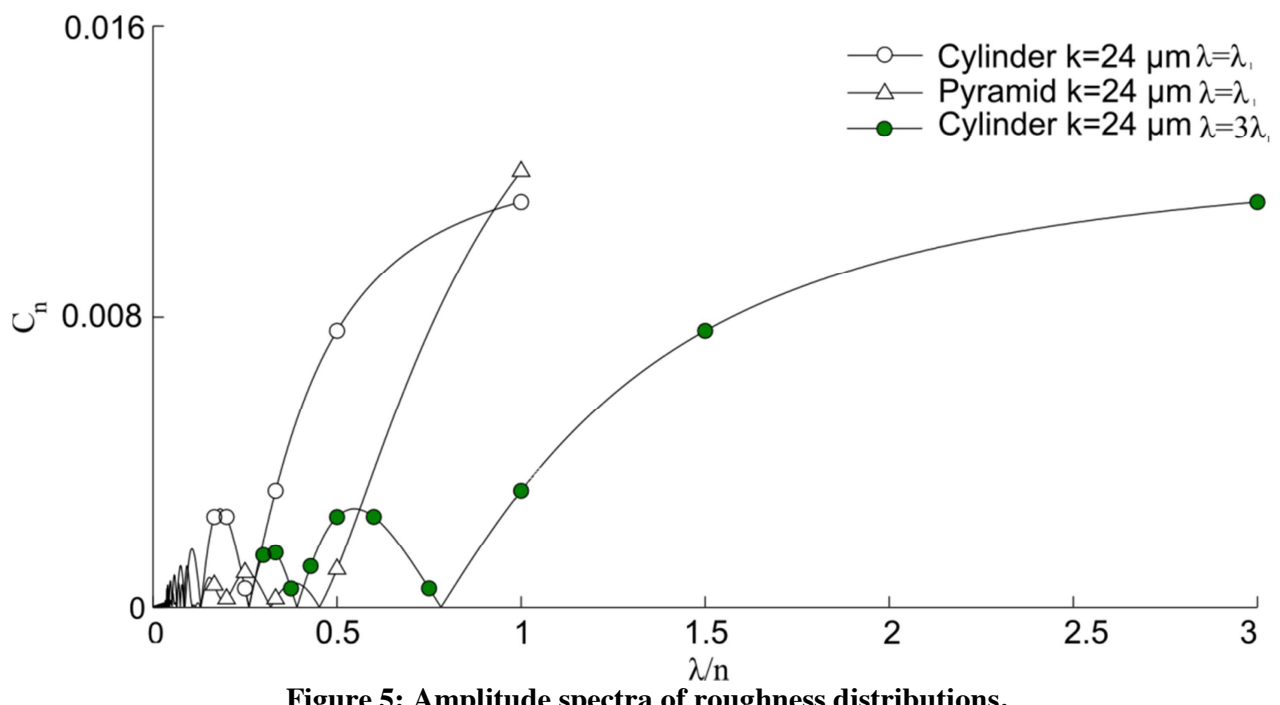

Figure 5: Amplitude spectra of roughness distributions.

\section{Wind tunnel facility}

All experiments are conducted in the UK National Low Turbulence wind tunnel at City University London. This closed circuit tunnel is equipped with a test section of $3 \mathrm{ft} \times 3 \mathrm{ft} \times 6 \mathrm{ft}$ and can be operated in the velocity range 0.5 $45 \mathrm{~m} / \mathrm{s}$. The tunnel has very low freestream turbulence levels, which makes it an excellent facility to study boundary layer transition following the natural transition path. The tunnel turbulence intensity measured in an empty tunnel is $0.006 \%$ of the free-stream velocity within the frequency range $4 \mathrm{~Hz}-4 \mathrm{kHz}$ at a freestream velocity of nominally 18 $\mathrm{m} / \mathrm{s}$.

\section{Experimental techniques and data acquisition}

Constant temperature hot wire anemometry (CTA) is chosen as main experimental technique since it allows for measurements with both high-spatial and temporal resolutions. This is essential in the current study where the boundary layer is expected to be thin and the disturbances small. With this technique, the flow velocity and fluctuations are measured with a Dantec hot-wire sensor (with effective sensing length $l \approx 1 \mathrm{~mm}$ and diameter $d \approx 5$ $\mu \mathrm{m})$. The analog signals (i.e. pressure transducer, hot wire sensor, temperature sensor, etc.) are digitized using an NI PXI6143 card in combination with a DAQ-BNC2021 data acquisition module. These are coordinated via a NI PXI Chassis, which is operated by an in-house data acquisition software written in LabVIEW. The sample duration is varied across the different measurements, however, the sample frequency is fixed at $20 \mathrm{kHz}$. Low-pass and highpass filters are controlled via a filter and set to $10 \mathrm{KHz}$ and $2 \mathrm{~Hz}$, respectively. The voltage to velocity conversion for the mean velocity, U, and fluctuating components, u', is obtained via King's law fit, as highlighted in Bruun 39. To minimize the calibration error, the probes are calibrated in place in the velocity range $0.5 \mathrm{~m} / \mathrm{s} \leq \mathrm{Q} \leq 18 \mathrm{~m} / \mathrm{s}$ against a Pitot-static tube connected to a SETRA manometer.

To ensure that the hot wire does not come in contact with the wall, a laser positioning system is connected to the traverse stand. The laser measures the distance to the wall at each hot wire measurement location. In this way, the relative distance of the hot wire to the wall is corrected for a possible inclination of the plate as well as undulations due to the machining.

The pressure on the flat plate is measured via classic static pressure ports $(0.5 \mathrm{~mm}$ in diameter $)$ embedded in the plate surface and connected to a Furness (FCO560) pressure transducer. To verify that the flow is two dimensional three rows of pressure port, aligned in the streamwise direction are installed. 


\section{E. Coordinate systems and data analysis}

Hot wire measurements are carried out with respect to the traverse and model coordinate system denoted by $\mathrm{X}, \mathrm{Y}, \mathrm{Z}$ and $x, y, z$ respectively (Fig. 6). The hot wire is aligned with the $\mathrm{x}$-direction measuring the stream wise velocity $u$.

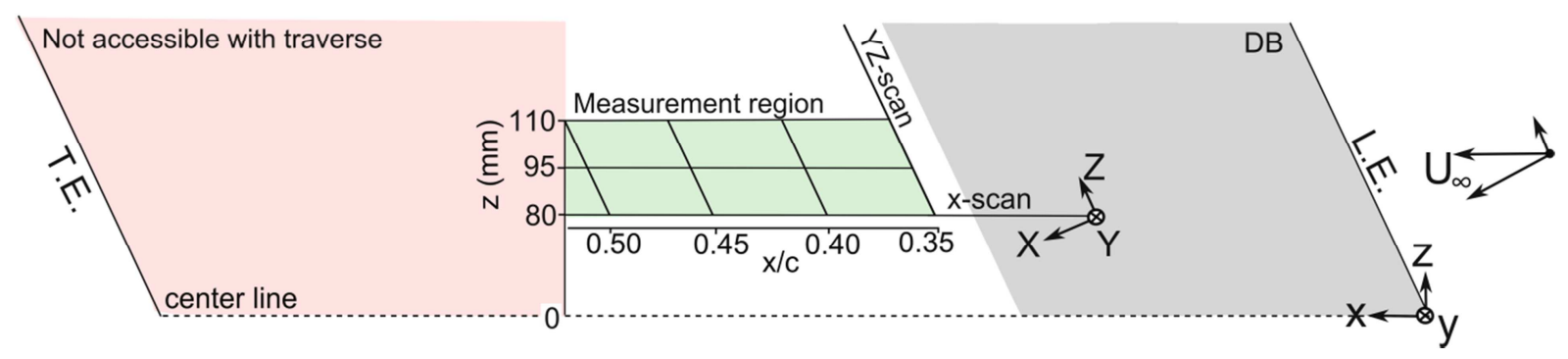

Figure 6: Coordinate systems used in measurements together with the hot wire scans which are carried out.

There are two types of hot wire scans carried out, which give different information about the flow. Firstly, x-scans are carried out at one normal location $(\mathrm{y}=1 \mathrm{~mm})$ and several spanwise locations with $\Delta x / c=0.003$ and an acquisition time of 5 seconds. From the time signals of the $\mathrm{x}$ scans the appearance of high-frequency bursts are detected which indicates the onset of the secondary instability. The algorithm to define the bursts is shown in Figure 7. First, a smooth signal is subtracted from the raw signal. After that, a threshold determines if a burst is present. Throughout all experiments the threshold is kept constant. Even though the location where the burst appears can not be defined as the actual transition location, these are closely related, since in previous research it is found that transition takes place a few percent after the secondary instability appears ${ }^{3}$.
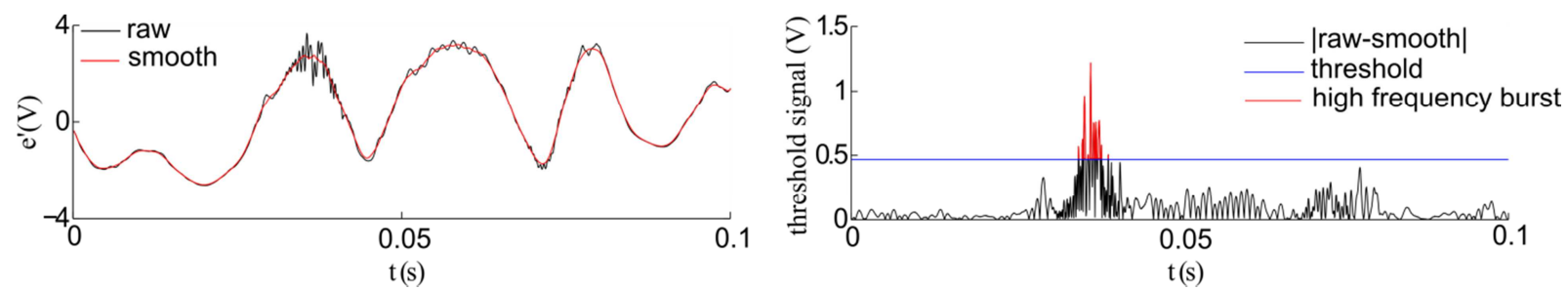

Figure 7: Definition of bursts of high frequency content in a signal.

Secondly, YZ-scans are carried out at four different $x / c$ locations depending on the case in examination. In these scans about 40 boundary layer profiles are taken parallel to the leading edge with a resolution of $\Delta Z=1 \mathrm{~mm}$. From this high-resolution data, the development of the stationary and travelling crossflow waves is determined. The data analysis consists of several pre-processing and calculation steps. Preprocessing starts with a linear interpolation of the measurement points in the near wall to define the exact location of the wall. Next the shifted and scattered data is interpolated to create a uniform grid in $\mathrm{Y}$ and $\mathrm{Z}$. From the interpolated grids the growth of a stationary or travelling instability a mode shape is determined with:

$$
Q(y)=r m s\left(\frac{q-\bar{q}}{U_{e}}\right) .
$$

Here $\bar{q}$ denotes the value of $q$ averaged over $\mathrm{Z}$ and $U_{e}$ the mean velocity at the edge of the boundary layer. Here $q$ can be the mean velocity $U$ or the fluctuating velocity $u_{r m s}^{\prime}$. The amplitude of mode shape $Q$, is then determined as $A=\max (Q)$. 
From the filtered velocity signal power spectra are calculated. First the signal is divided in blocks, after which a Hanning window function is applied. Next the power spectra density of each block is calculated following the Welch method ${ }^{40}$. Finally, an average of all the blocks results in the final power spectra. A spatial power spectra is also calculated for the mean velocity fluctuations at a constant wall-normal location.

From the analysis of the power spectra, the frequencies of the travelling instabilities were identified. The signal was band passed filtered in these frequency ranges by applying an infinite impulse response filter. From the filtered signals $r m s$ values were calculated with $u_{r m s}^{\prime}=e_{r m s}^{\prime} / d E / D U$. Where $E$ is the mean of the voltage signal $U$ the mean velocity and $e_{r m s}^{\prime}$ the root mean square value of the filtered AC voltage signal. Finally, from the velocity fluctuation distributions mode shapes and amplitudes were calculated.

The bandpassed filtered velocity fluctuations distributions were correlated to the $D U / D Y$ and $D U / D Z$ gradient fields to identify how the instabilities are distributed with respect to the underlying mean flow. To do so the gradient fields were smoothened and a correlation coefficient $R$ was applied:

$$
R=\frac{\sum_{m} \sum_{n}\left(F_{m n}-\bar{F}\right)\left(G_{m n}-\bar{G}\right)}{\sqrt{\sum_{m} \sum_{n}\left(F_{m n}-\bar{F}\right)^{2} \sum_{m} \sum_{n}\left(G_{m n}-\bar{G}\right)^{2}}}
$$

where $F$ and $G$ are the velocity distributions which are correlated.

\section{Results and Discussion}

\section{A. Pressure measurements}

The measured pressure distribution is shown in Figure 8 and compared to pressure distributions from previous crossflow experiments ${ }^{15,17,31}$ favourable pressure gradient is present and extends well behind the displacement body (light gray in Figure 7). In the region up to $x / c \approx 0.2$ the pressure gradient in previous studies by Saric is a lot stronger than in the current experiment. This reduced pressure gradient might cause the crossflow waves to develop less strongly and rapidly. The gradient from $x / c \approx 0.2$ is similar to the pressure gradient used in previous studies, which indicates that the current setup is viable to study the development of the crossflow instability. The pressure on the upper part of the plate was uniform, and hence the infinite swept condition was met. For these reasons all hot-wire measurements are taken slightly above the centerline of the plate.

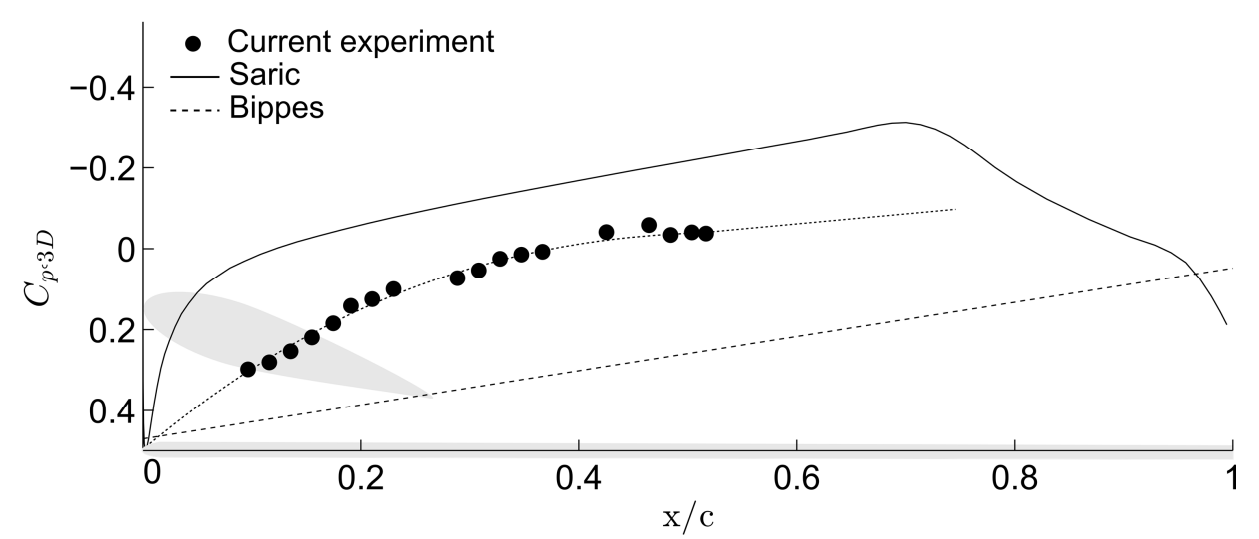

Figure 8: Experimental pressure measurements and comparison with pressure distributions from other crossflow experiments $^{15,17,31}$. 


\section{B. Linear stability analysis}

The measured pressure distribution was used in a complimentary Linear Stability Analysis (LSA) based on the experimental model. In order to do so the experimental data was fitted with a polynomial (dotted line in Figure 8) to obtain information about the pressure in the leading edge region. In Figure 9 the $\mathrm{N}$-factors for the stationary waves are shown together with the maximum growth of T-S waves. It is concluded that the flow over the model is crossflow dominated and furthermore the $11.5 \mathrm{~mm}$ mode gives maximum growth in the measurement region. It is also found that waves with a wavelength of $12-13 \mathrm{~mm}$ are expected to significantly grow.

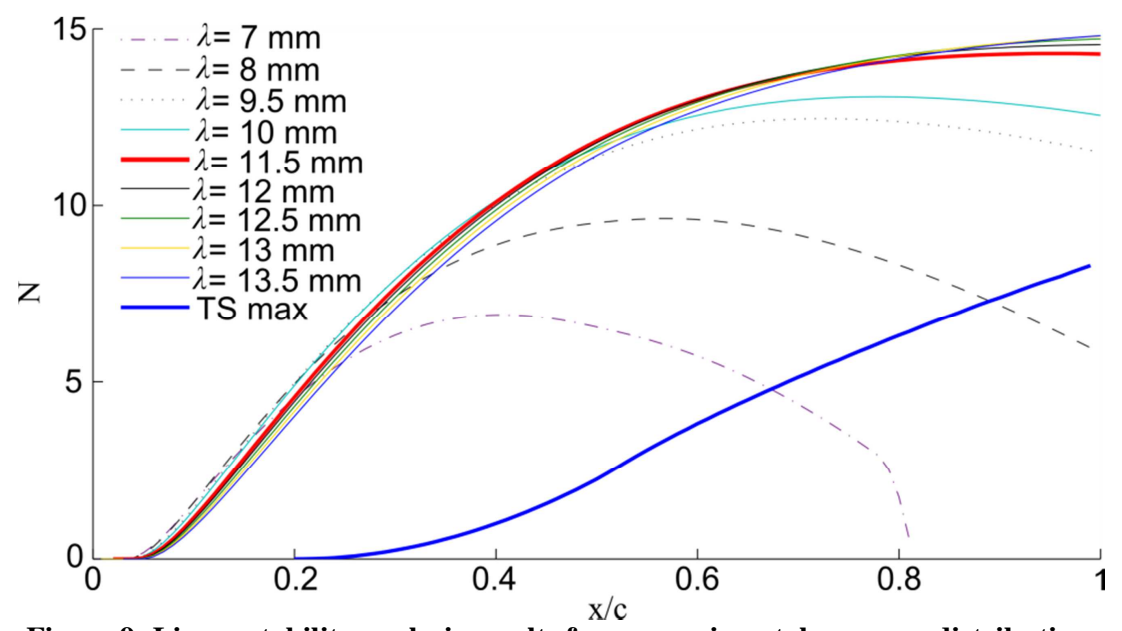

Figure 9: Linear stability analysis results from experimental pressure distribution.

\section{Hot wire measurements}

The mean flow distribution is shown for the first measurement location, at $x / c=0.35$ (Fig. 10). The roughness elements have a large influence on the mean flow and cause the stationary crossflow waves to grow more strongly when compared to the flow without roughness. The velocity profiles show that the flow is still fully laminar at this location and additionally the mean profile does not show large distortions (Fig. 10b). From the stationary crossflow mode shapes it is concluded that the pyramidal roughness distributions results in a higher amplitude of the stationary crossflow mode compared to the cylindrical roughness distributions (see Figures 3 and 4). This is believed to be due to the stronger forcing at the critical wavelength $\lambda_{\text {crit }}$, as demonstrated in Figure 5. Furthermore, a second lobe seems to appear around $\mathrm{Y}=1.5 \mathrm{~mm}$ in the stationary mode shape for the pyramidal elements, which indicates the start of non-linear effects ${ }^{25}$.

It is clear that the flow becomes more organized by placing the roughness elements (Fig. 10a), however, a very high level of uniformity as shown in previous investigations ${ }^{24,31}$ has not been achieved. The reason for this is yet unclear but might be attributed to the stronger favourable pressure gradient in other studies. 


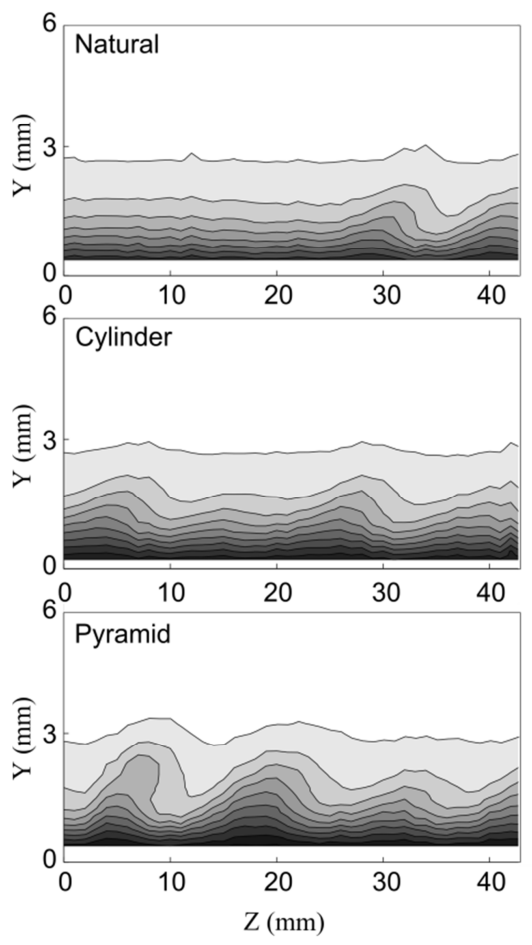

(a)
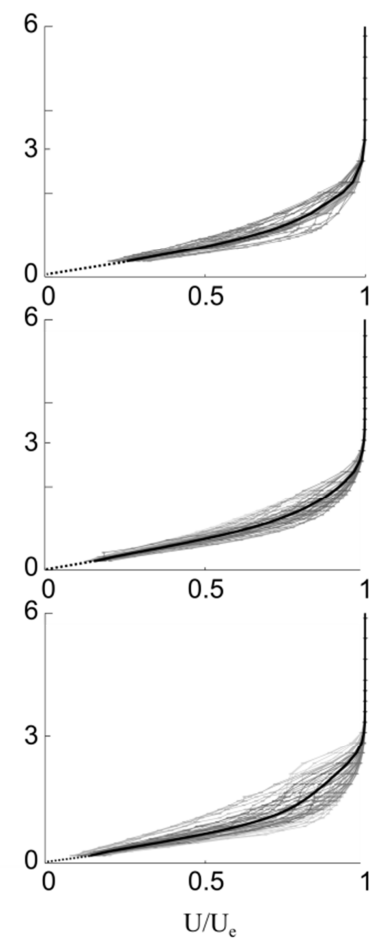

(b)
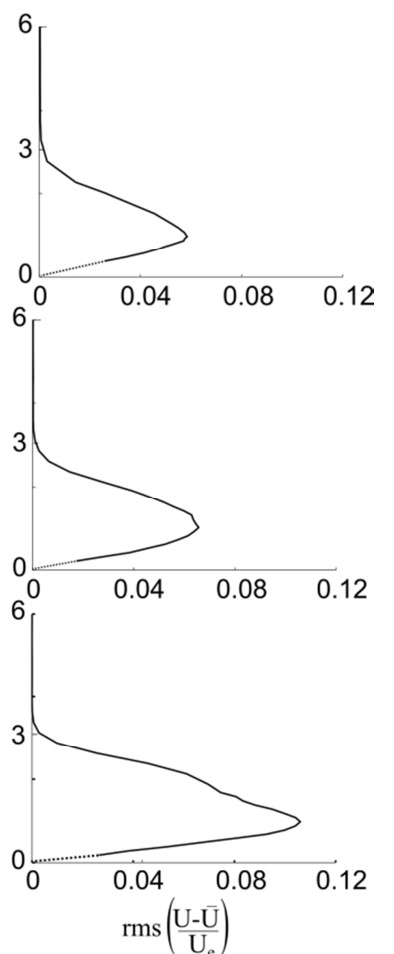

(c)

Figure 10: Mean flow distributions (a), velocity distributions (b) and stationary mode shapes (c) at $\mathrm{x} / \mathrm{c}=0.35$.

To show the nonlinearity of the flow, the development of the stationary crossflow mode shapes are shown in more detail in Figure 11. The nonlinear effects appear earlier for the pyramidal elements, where the second lobe grows up to $x / c=0.45$. At this location the mode shapes of both roughness distributions look very similar. At the final measurement location the pyramidal mode shape breaks down. A similar effect has been shown in previous studies when the roughness height was increased. First there is a stronger response for the larger roughness elements but when the vortices saturate, the mode shapes become similar to each other. In previous studies it is found that the nonlinear growth of the stationary crossflow waves is caused by the interaction of $\lambda / n$ modes ${ }^{24,41}$ Here it is shown that the frequency content of the roughness distribution at $\lambda / n$ modes has no effect on this process: it is only the stronger forcing at $\lambda$ that dominates the response of the flow. 

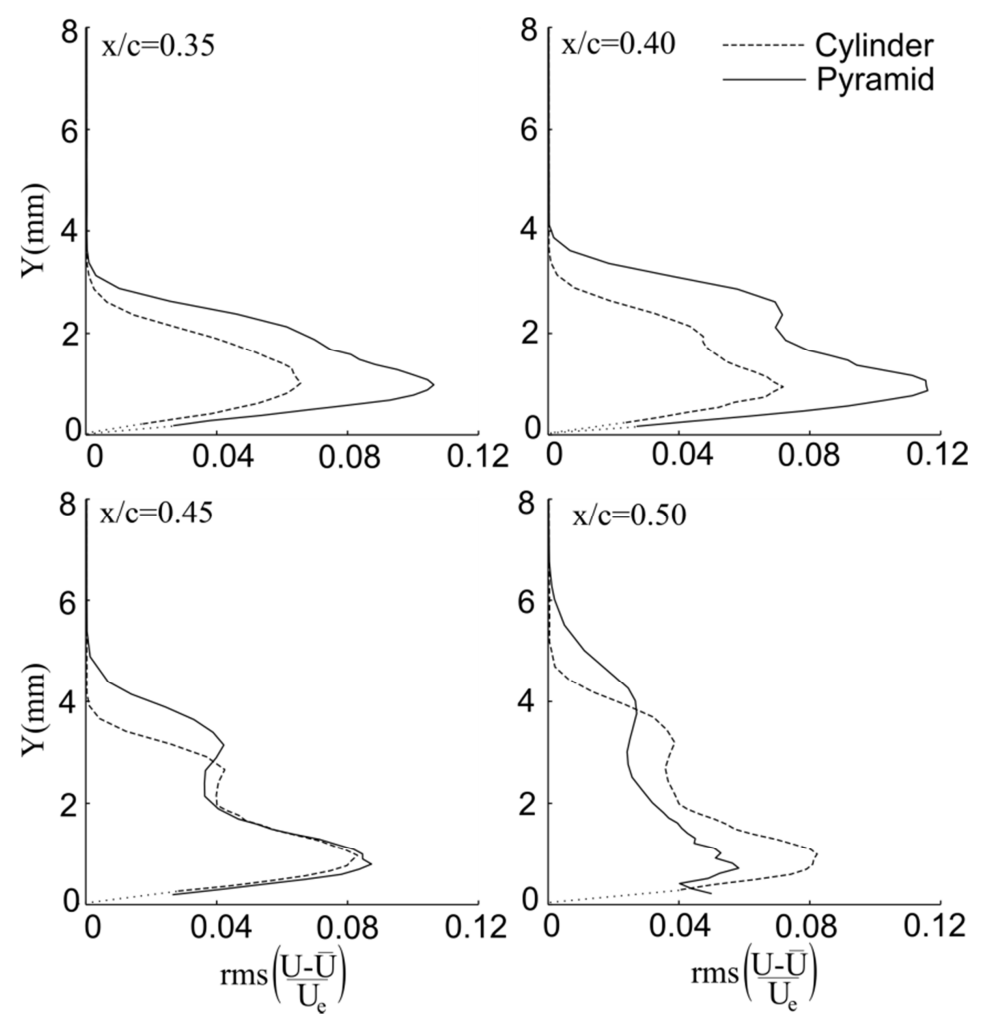

Figure 11: Development of stationary mode shape for $k=24 \mu \mathrm{m}$.

To understand which modes are amplified in the mean flow, spatial spectra are computed at the y location where the maximum in the mode shape occurs (based on Figure 11). The results are shown for selected wavelengths in Figure 12. The accuracies are computed following Reibert ${ }^{24}$. It is shown that at the first two measurement locations the 11.5 $\mathrm{mm}$ mode is more strongly forced for the pyramidal roughness distribution. Downstream the $11.5 \mathrm{~mm}$ mode breaks down, while the spectral energy content of the larger wavelengths $(\lambda>13.5 \mathrm{~mm})$ increases. This suggests that the vortices break down and merge into larger structures. At the $\lambda / 2$ mode $(\lambda=5.7 \mathrm{~mm})$ very little activity is observed. Again the spatial spectrum suggests that the stronger response of the flow at $\lambda$ is due to the shape of the roughness distribution.

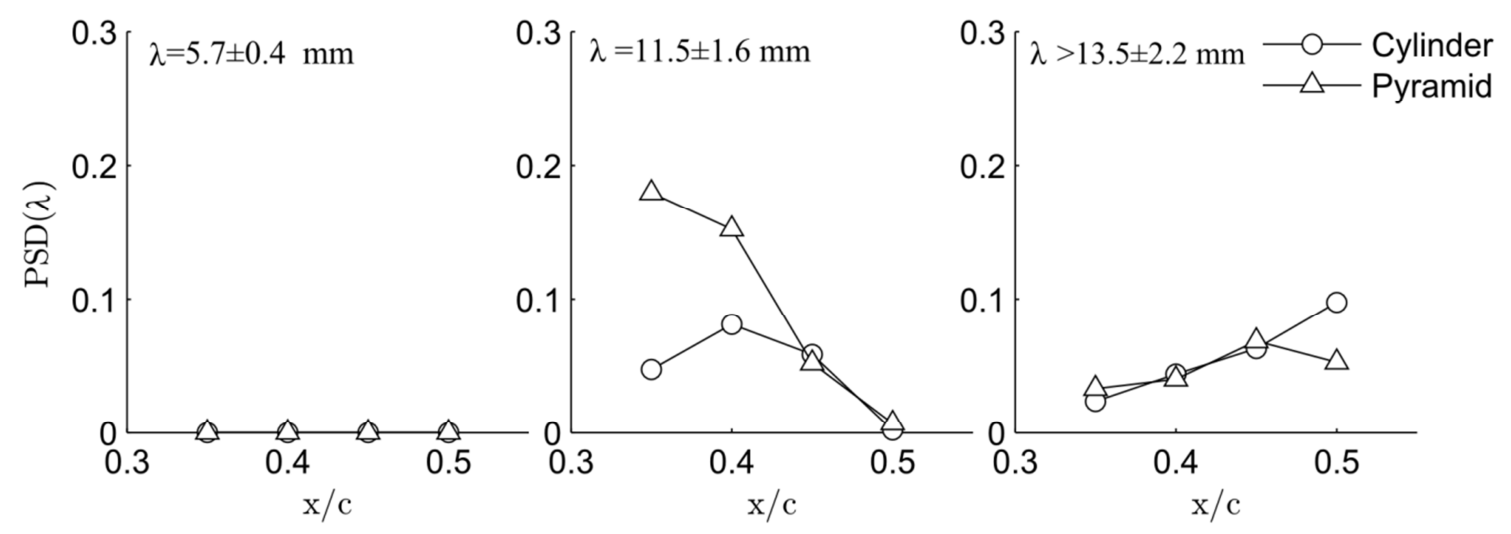

Figure 12: Maximum amplitude of spatial power spectra of mean velocity $U$ at several wavelengths $\lambda$. 
Next to the stationary instabilities the travelling instabilities are studied in more detail. In Figure 13 the normalized $\mathrm{u}_{\mathrm{rms}}^{\prime}$ is shown for the first and last measurement locations. The vortical structures at the first measurement location have a wavelength of about $\lambda / 2$. Similar findings were found in previous computational and experimental studies in low turbulence environments ${ }^{10}$. It is suggested in these studies that there is a strong interaction between the travelling waves and the $\lambda / 2$ mode of the stationary wave. The amplitude spectra at $\lambda$ and $\lambda / 2$ show again increased energy content for the pyramidal elements (Fig. 14). The development of the mode shapes shows a similar behavior as for the stationary wave. The initial amplitude is higher for the pyramidal elements after which the mode shapes become almost identical (Fig. 15). Again this analysis of $\mathrm{u}_{\mathrm{rms}}^{\prime}$ confirms that the frequency content of the roughness distribution at $\lambda$ dominates the flow, while the content at $\lambda / n$ seems irrelevant.
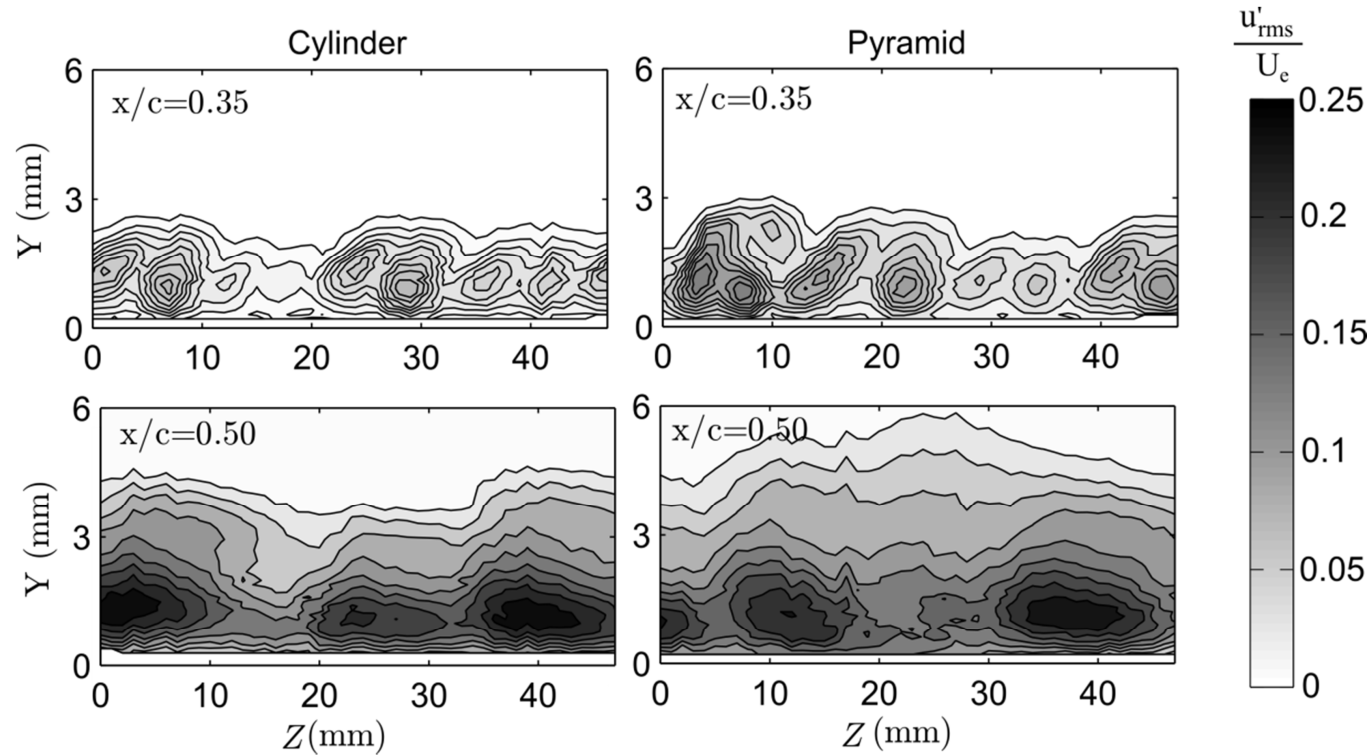

Figure 13: Distribution of normalized velocity fluctuations $\mathbf{u}^{\prime}{ }_{\mathrm{rms}} / \mathbf{U}_{\mathrm{e}}$.

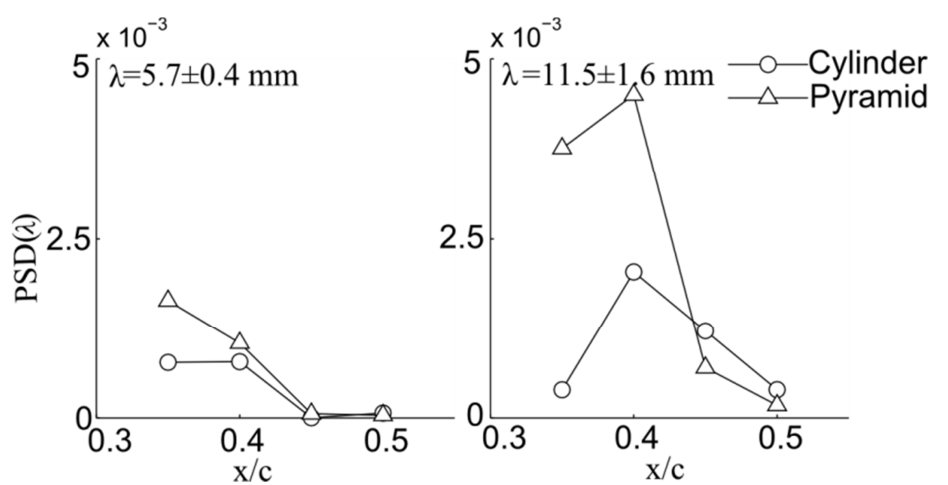

Figure 14 : Amplitude of spatial power spectra of mean velocity $\mathbf{u}_{\mathrm{rms}}{ }$ at several wavelengths $\lambda$. 
Next the spectra at the first and last measurement location were inspected (Fig. 16). These are spectra as computed for just one spanwise location. To analyze the spanwise and wall-normal distributions of the instability, two regions of interest are identified. A low-frequency region in the range $200-250 \mathrm{~Hz}$ and a high-frequency region from 900-3000 Hz. The voltage signal is bandpassed filtered for these two regions and the velocity fluctuations are calculated for each measurement point.
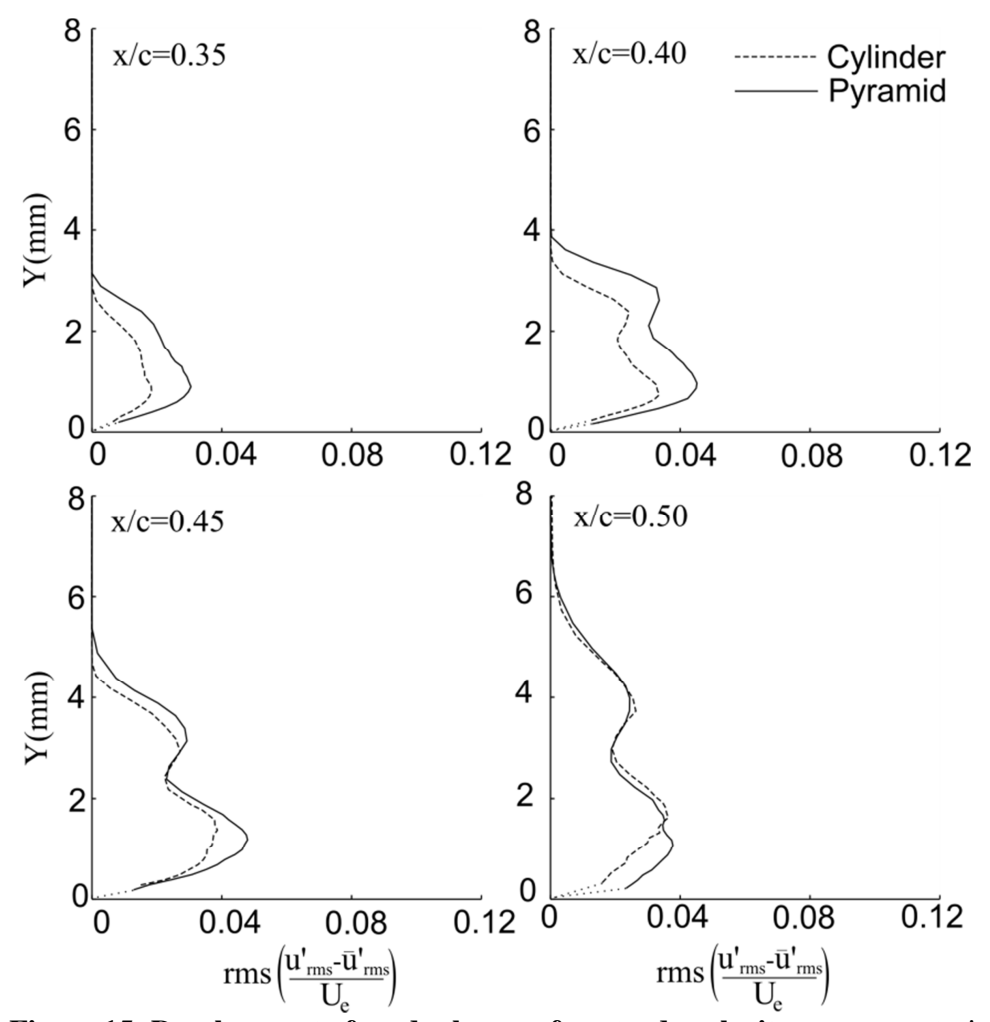

Figure 15: Development of mode shapes of unsteady velocity component $u^{\prime}$.

In Figure 17 the distribution of the velocity fluctuations is shown for $200-250 \mathrm{~Hz}$ for the first two measurement locations. In the first location, clear vortical structures are found for both roughness distributions. At the last measurement location large structures are highlighted and the flow is highly three-dimensional. This ties in well with the power spectra in Figure 16, where the peak at 200-250 Hz disappears. The fluctuations and structure are similar for both roughness distributions.

For the high-frequency fluctuations $(900-3000 \mathrm{~Hz})$, no coherent structure is observed at the first measurement location (Fig. 18). At the last location there are large structures for both roughness distributions and again the flow is highly three-dimensional. The fluctuations are signigicantly stronger for the pyramid but due to the threedimensionality it is difficult to relate this directly to the difference in shape of both distributions. 


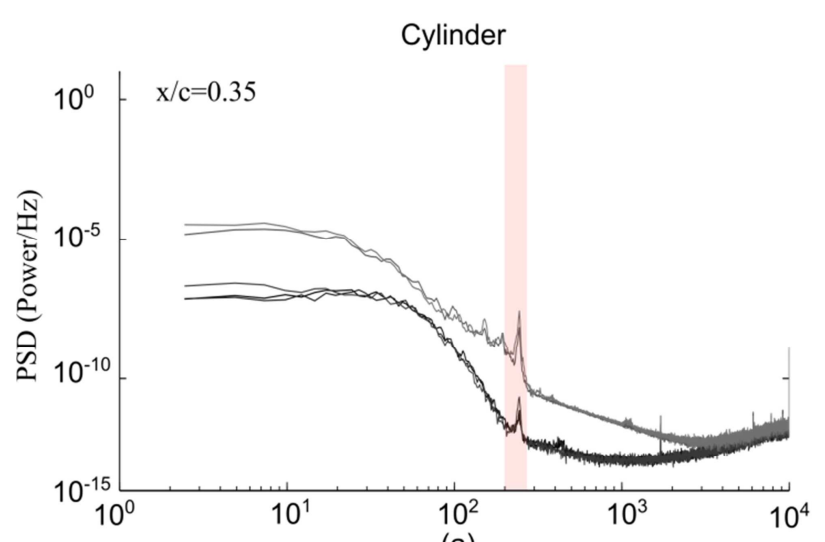

(a)

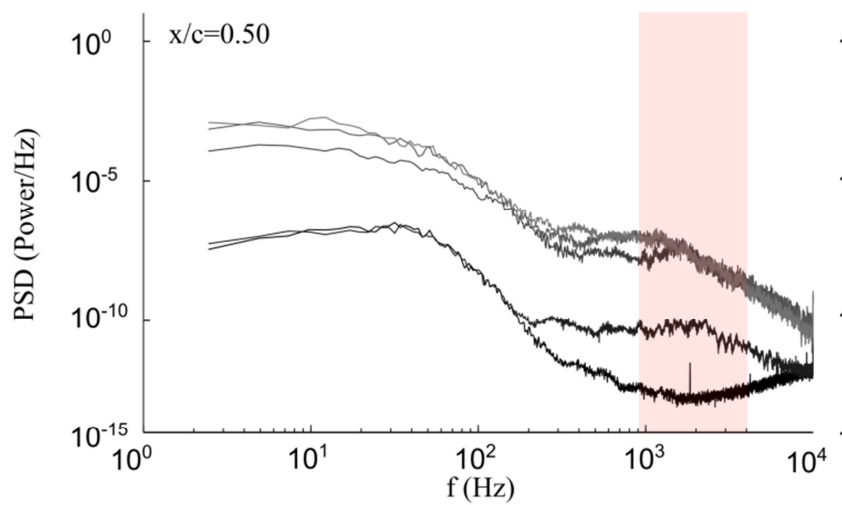

(c)
Pyramid

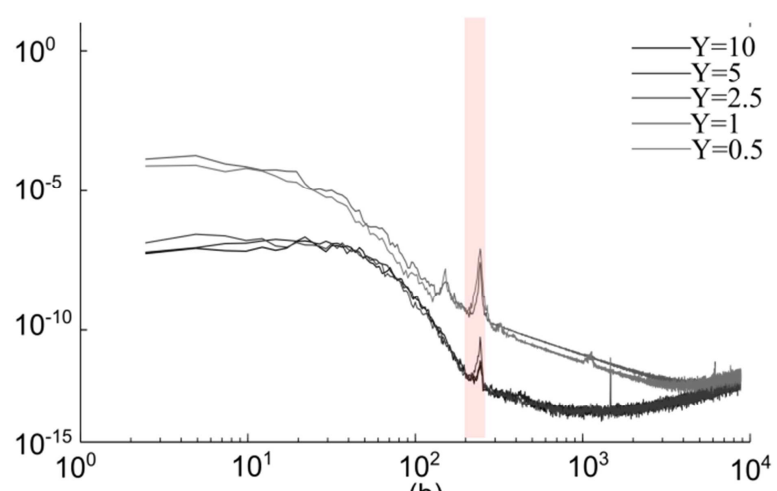

(b)

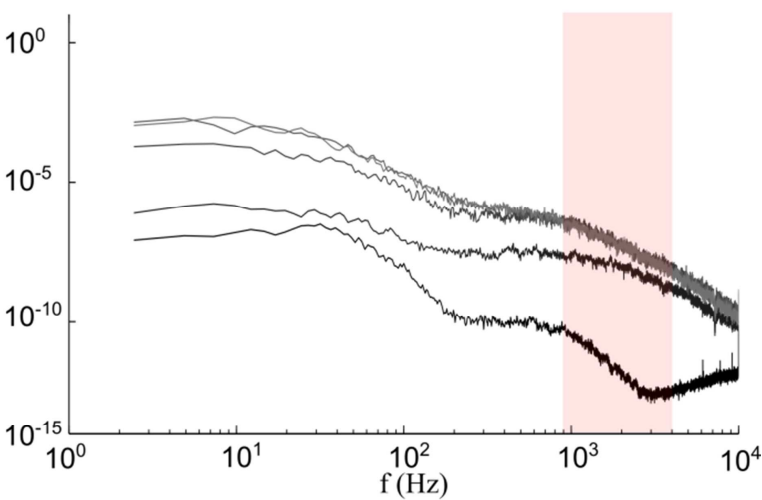

(d)

Figure 16: Power spectra at selected Y normal locations for $x / c=0.35$ (a and $b)$ and $x / c=0.50$ (c and d). Highlighted in red the regions object of further analysis.
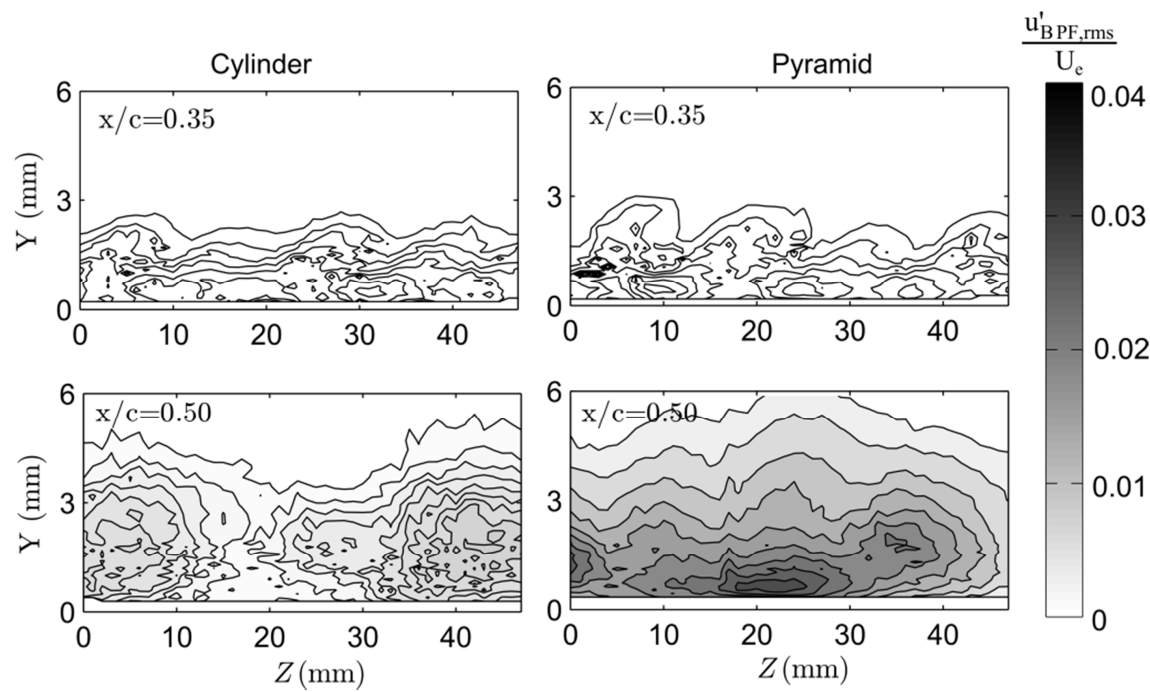

Figure 17: Distribution of normalized velocity fluctuations between $200-250 \mathrm{~Hz}$. 
The wavelengths for the vortices at the low frequency $(200-250 \mathrm{~Hz})$ velocity fluctuations is about $\lambda$ (Figure 17), which indicates that there is a strong interaction in between travelling and stationary waves. To quantify this interaction, the velocity fluctuation fields were correlated with the gradient fields (DU/DY and $D U / D Z)$. The gradient fields highlight shear layers in the flow, which are known to cause secondary instabilities ${ }^{11}$. In Figure 19 the velocity fluctuation field for $200-250 \mathrm{~Hz}$ and $900-3000 \mathrm{~Hz}$ is overlapped on the underlying mean flow fields. Because of length restrictions only a part of the velocity fields is shown but the correlation coefficient $R$ indicates the correlation between the entire velocity fields.

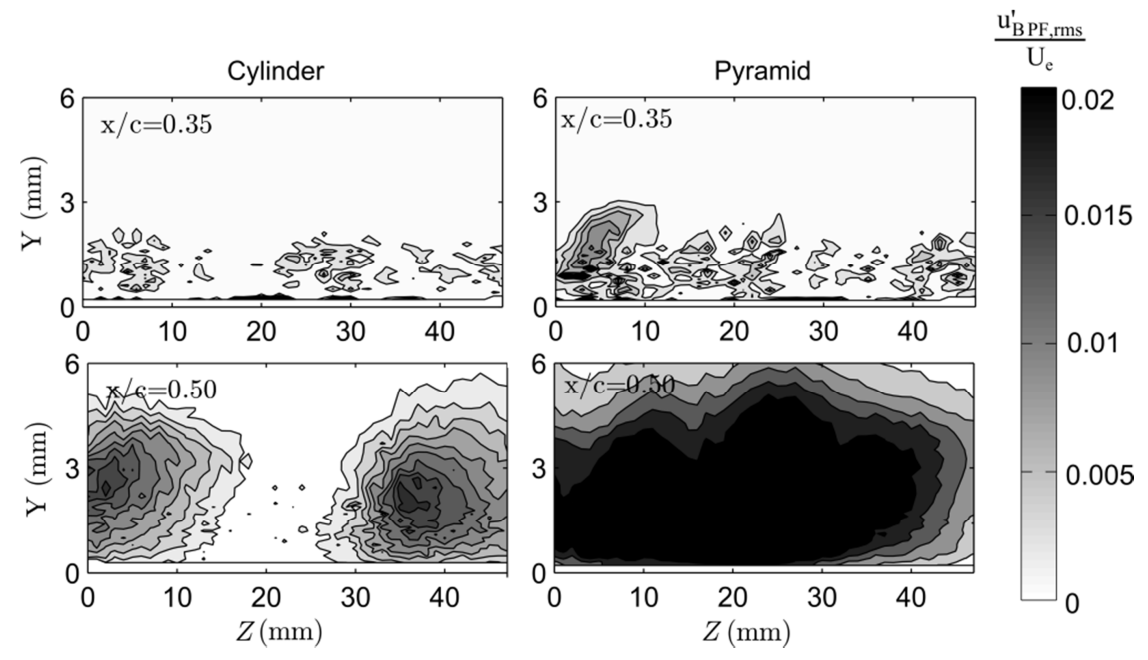

Figure 18: Distribution of normalized velocity fluctuations between $900-3000 \mathrm{~Hz}$.

The 200-250 Hz instability is strongly correlated with the DU/DY field at the first location (Fig. 19) . At the last location no strong correlation is found. This indicates that this instability is caused by an interaction with the shear layer caused by the stationary waves ${ }^{11}$.

The 900-3000 Hz instability is stronger correlated to the $D U / D Z$ field at the first location (Fig. 20). For the pyramid this correlation is similar at the last location while for the cylinder it decreases. These results suggest that the highfrequency instability is caused by a shear layer in the $D U / D Z$ field. In previous research two different types of instabilities were reported: Type I (caused by a shear layer in DU/DZ) and Type II (caused by a shear layer in $D U / D Y$ ) secondary instabilities were identified ${ }^{9,11,41}$. In this analysis the high-frequency region is not analyzed in separate frequency bands, and therefore separate modes could not be distinguished.

The trends for the pyramid and cylinder are very similar in this travelling instabilitiy analysis, so no clear relationship between this instability and the frequency content of the roughness contribution can me made. However, as far as the authors are concerned, a correlation analysis such as shown here, has not been utilized in previous studies to identify the interactions between instabilities and is expected to be a useful tool in future investigations. 

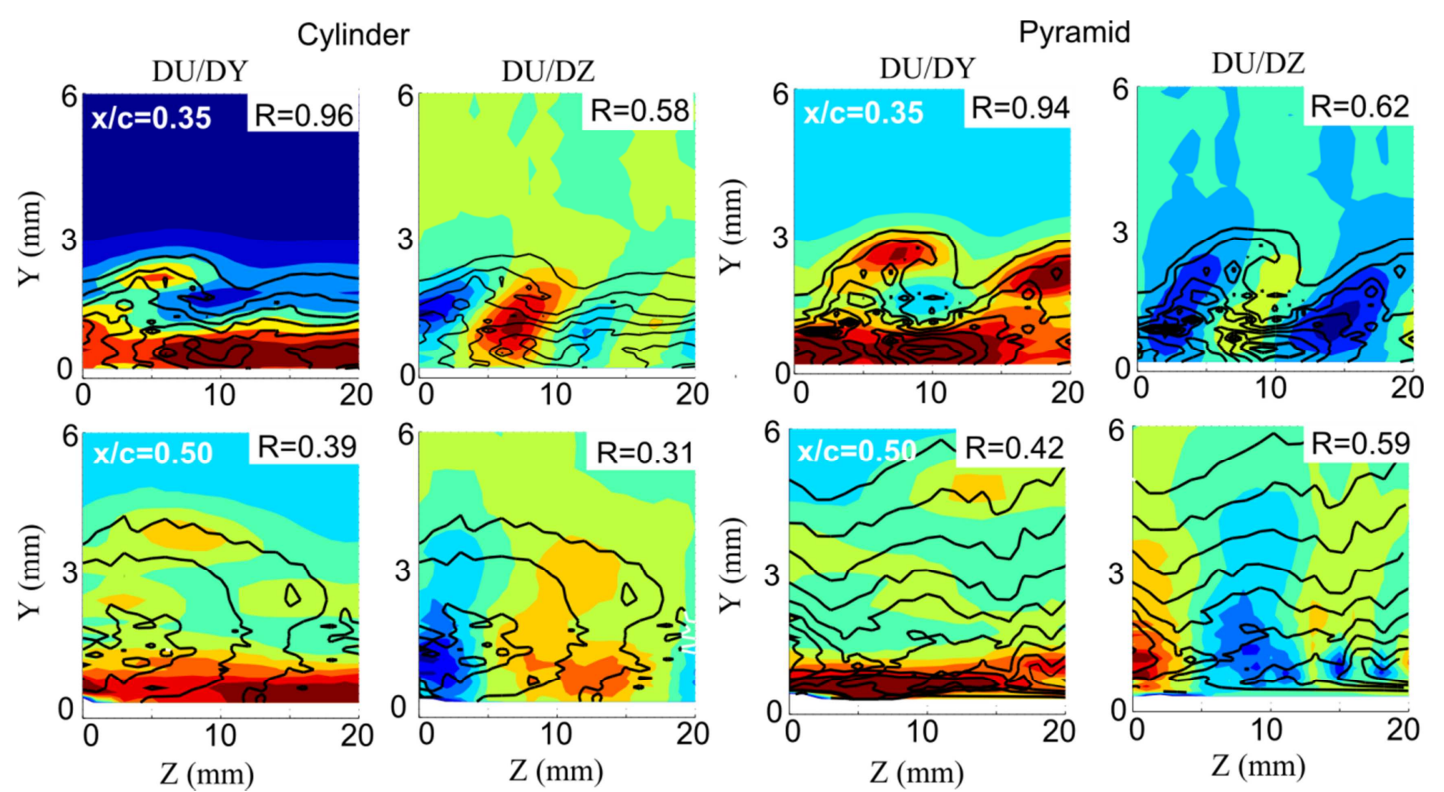

Figure 19: Correlation between 200-250 Hz velocity fluctuations (black lines) and underlying mean flow (coloured contours).
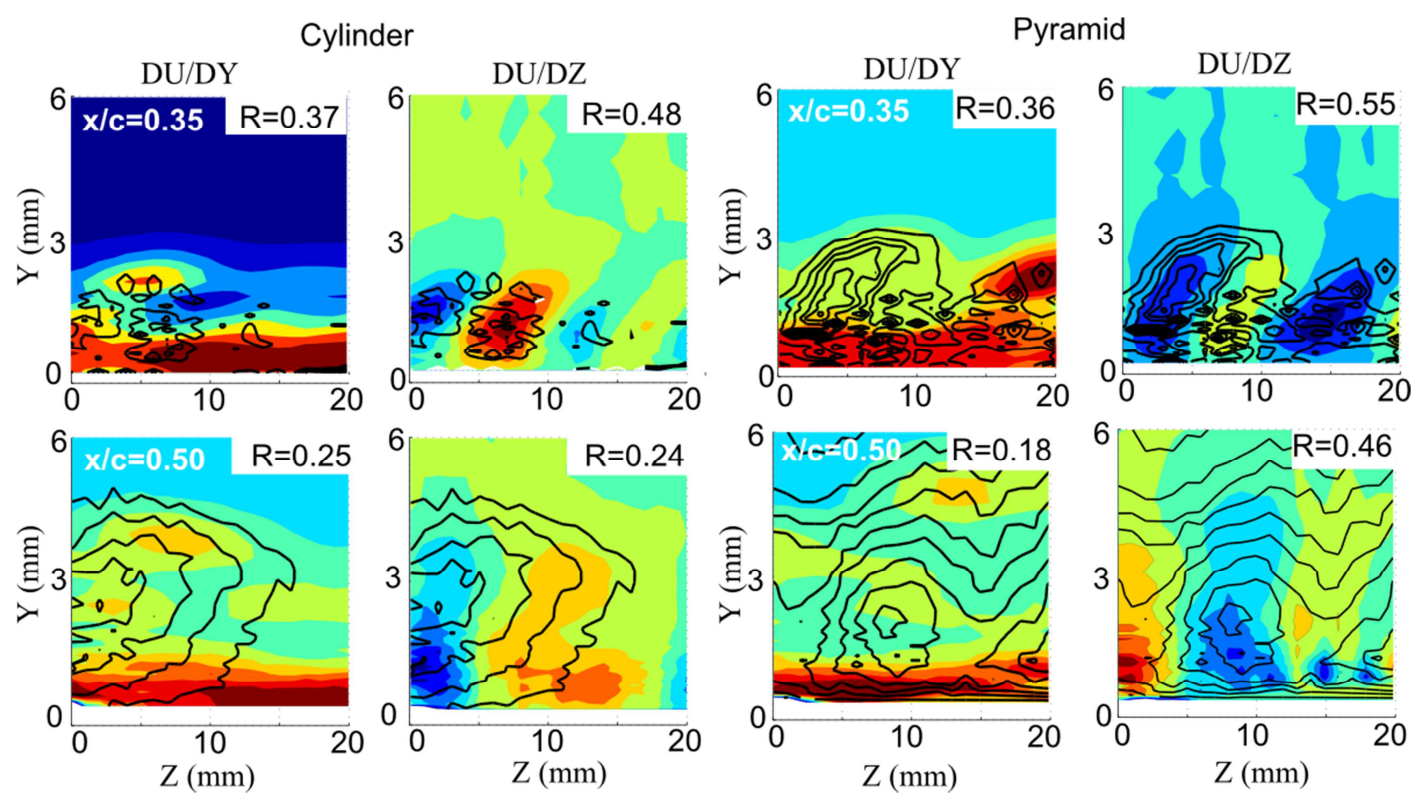

Figure 20: Correlation between 900-3000 Hz velocity fluctuations (black lines) and underlying mean flow (colored contours).

From the YZ-scans it seems that the strength and growth of the secondary instabilities is influenced by the roughness shape (see Figure 18). However the onset of the secondary instability, which is strongly tied with the transition location, cannot be detected from the YZ-scans. Therefore the x-scans are analyzed in more detail. The results show that the high-frequency bursts appear earlier when roughness elements are placed on the surface of the plate (Fig. 21). There seems to be a small difference between the cylindrical and pyramidal roughness elements. However, due to the large uncertainty it is does not seem to be significant. Since transition takes place a few percent after the onset of the secondary instability, it indicates that there is no effect on transition. In previous research it has been found that when the roughness height is increased transition does not move forward if the saturation amplitude is reached. When the roughness height is increased the forcing at $\lambda$ and $\lambda / n$ is increased. 


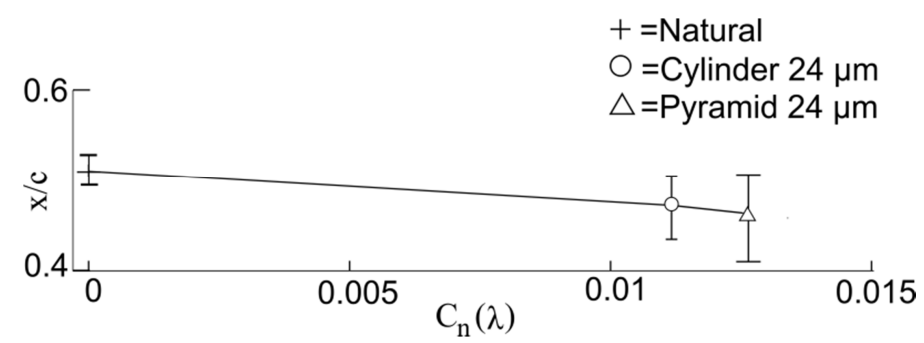

Figure 21: Onset of high-frequency bursts against the forcing of the roughness distributions at $\lambda$ (see Figure 5).

Reibert $^{24}$ found that when the flow is forced with roughness elements spaced at $3 \lambda$ transition does move forward. In the current experiment it is found that stronger forcing at $\lambda$ and weaker forcing at the harmonics at $\lambda / n$ does not seem to influence the transition location strongly; however more detailed measurements should confirm this. All the results presented here and of previous studies indicate that the forcing of $\lambda / n$ via the roughness distribution does not have a large effect on the development of the crossflow instability if $\lambda$ is chosen to be the most unstable wavelength following linear stability analysis.

\section{Conclusion and future work}

An experimental investigation on the influence of the frequency content of the roughness distribution on the development and growth of crossflow vortices has been carried out. It is demonstrated that the stronger forcing via roughness distribution at the most unstable wavelength $\lambda$ leads to a stronger response for the stationary crossflow waves, which is in line with Fourier analysis and previous experiments. The results from the experiment indicate that stronger forcing at the critical wavelength dominates the development of the stationary and travelling crossflow waves flow, even if the harmonics of the critical wavelength are strongly damped. It is found that the onset of highfrequency secondary instability is not significantly influenced by placing the roughness elements close to the neutral stability point however more detailed measurements are needed to confirm this.

In further investigations larger roughness elements will be tested in order to see if the same trends hold. Next, the influence on roughness on the interaction between the travelling and stationary waves will be further investigated.

\section{Acknowledgments}

This work was funded by the Engineering and Physical Sciences Research Council under the LFC-UK Programme Grant, ref EP/I037946/1.

\section{References}

\footnotetext{
${ }^{1}$ Morkovin, M.V., "On the many faces of transition," Viscous drag reduction, edited by C.S. Wells, Springer US, 1969, pp. 1-31.

${ }^{2}$ Kachanov, Y.S., "Physical mechanisms of laminar-boundary-layer transition," Annual Review of Fluid Mechanics, Vol. 26, No. 1, 1994, pp. 411-482.

${ }^{3}$ Saric, W.S., Reed, H.L., and White, E.B., "Stability and transition of three-dimensional boundary layers," Annual Review of Fluid Mechanics, Vol. 35, No. 1, 2003, pp. 413-440.

${ }^{4}$ Atkin, C., "Laminar Flow Control: Leap or Creep?," 38th Fluid Dynamics Conference and Exhibit, AIAA, Seattle, WN, 2008.

${ }^{5}$ Gray, W.E., "The effect of wing sweep on laminar flow," Royal Aircraft Establishment TM Aero. 255, 1952

${ }^{6}$ Kundu, P.K. and Cohen, I.M., Fluid Mechanics,Elsevier, $4^{\text {th }}$ ed.,Academic Press,2008.

${ }^{7}$ Gregory, N., Stuart, J., and Walker, W., "On the stability of three-dimensional boundary layers with application to the flow due to a rotating disk," Philosophical Transactions of the Royal Society A, Vol. 248, No. 943, 1955, pp. 155-199.

${ }^{8}$ Poll, D.I.A., "Some observations of the transition process on the windward face of a long yawed cylinder," Journal of Fluid Mechanics, Vol. 150, No. 1, 1985, pp. 329-356.

${ }^{9}$ Kohama, Y., "Some expectations on the mechanism of cross-flow instability in a swept wing flow," Acta Mechanica, Vol. 66 pp. 21-38, 1987.

${ }^{10}$ Malik, M.R., Li, F. and Chang, C.L., "Nonlinear crossflow disturbances and secondary instabilities in swept-wing boundary Layers," Proceedings of the IUTAM Symposium, Manchester, U.K., 1996.

${ }^{11}$ White, E.B. and Saric, W.S., "Secondary instability of crossflow vortices," Journal of Fluid Mechanics, Vol. 525, 200 pp.275-308.

${ }^{12}$ Tollmien, W., "Über die Entstehung der Turbulenz. 1. Mitteilung," Nachrichten von der Gesellschaft der Wissenschaften zu
} 
Göttingen, Mathematisch-Physikalische Klasse, 1928.

${ }^{13}$ Schlichting, H., "Berechnung der Anfachung kleiner Störungen bei der Plattenströmung," Zeitschrift für Angewandte Mathematik und Mechanik, Vol. 13, 1933, pp. 171-174.

${ }^{14}$ Malik, M., and Poll, D., "Effect of curvature on three-dimensional boundary-layer stability," AIAA Journal, Vol. 23, No. 9, 1985, pp. 1362-1369.

${ }^{15}$ Bippes, H., and Müller, B., "Disturbance growth in an unstable three-dimensional boundary layer," Numerical and Physical Aspects of Aerodynamic Flows IV, edited by T. Cebeci, Springer Berlin Heidelberg, Berlin, 1990, pp. 345-358.

${ }^{16}$ Bippes, H., "Instability features appearing on swept wing configurations," Laminar-Turbulent Transition, edited by D. Arnal and R. Michel, Springer, 1990, pp. 419-430.

${ }^{17}$ Deyhle, H., and Bippes, H., "Disturbance growth in an unstable three-dimensional boundary layer and its dependence on environmental conditions," Journal of Fluid Mechanics, Vol. 316, No. 73, 1996, pp. 114.

${ }^{18}$ Schrader, L., Brandt, L., and Henningson, D.S., "Receptivity mechanisms in three-dimensional boundary-layer flows," Journal of Fluid Mechanics, Vol. 618, No. 1, 2009, pp. 209-241.

${ }^{19}$ Kurian, T., Fransson, J.H., and Alfredsson, P.H., "Boundary layer receptivity to free-stream turbulence and surface roughness over a swept flat plate," Physics of Fluids, Vol. 23, No. 3, 2011.

${ }^{20}$ Downs R.S., "Environmental influences on crossflow instability," Ph.D. Dissertation, Aerospace Engineering, Texas A\&M University,College Station, TX, 2012.

${ }^{21}$ Riedel, H., and Sitzmann, M., "In-flight investigations of atmospheric turbulence," Aerospace Engineering, Vol. 2, No. 5, 1998, pp. 301-319.

${ }^{22}$ Choudhari, M., "Roughness-induced generation of crossflow vortices in three-dimensional boundary layers," Theoretical and Computational Fluid Dynamics, Vol. 6, No. 1, 1994, pp. 1-30.

${ }^{23}$ Bertolotti F. P., Receptivity of three-dimensional boundary-layers to localized wall roughness and suction. Phys. Fluids 12(7), pp. 1799-1809.2000.

${ }^{24}$ Reibert, M.S , "Nonlinear stability, saturation, and transition in crossflow-dominated boundary layers," Ph.D.

Dissertation,Arizona State University, 1996.

${ }^{25}$ Reibert, M.S., Saric, W.S., Carrillo Jr, R.B., "Experiments in nonlinear saturation of stationary crossflow vortices in a sweptwing boundary layer," 34th Aerospace Sciences Meeting and Exhibit, 1996.

${ }^{26}$ Saric, W.S., Carrillo Jr, R.B., and Reibert, M.S., "Leading-edge roughness as a transition control mechanism," 36th Aerospace Sciences Meeting and Exhibit, AIAA-98-0781, 1998.

27 Wassermann, P., and Kloker, M., "Mechanisms and passive control of crossflow-vortex-induced transition in a threedimensional boundary layer," Journal of Fluid Mechanics, Vol. 456, No. 4, 2002, pp. 49-84.

28 Saric, W.S., Carpenter, A.L., and Reed, H.L., "Passive control of transition in three-dimensional boundary layers, with emphasis on discrete roughness elements," Philosophical Transactions.Series A, Mathematical, Physical, and Engineering Sciences, Vol. 369, No. 1940, 2011, pp. 1352-1364.

${ }^{29}$ Eppink, J., "The interaction of crossflow instabilities and a backward facing step in swept boundary layer transition",Ph. D.

Dissertation, Mechanical Engineering Dept.,Tufts University, 2014.

30 Radeztsky, R.H., Reibert, M.S., and Saric, W.S., "Effect of isolated micron-sized roughness on transition in swept-wing flows," AIAA Journal, Vol. 37, No. 11, 1999, pp. 1370-1377.

${ }^{31}$ Hunt, L.E., and Saric, W.S., "Boundary-layer receptivity to three-dimensional roughness arrays on a swept-wing," 41 st AIAA Fluid Dynamics Conference and Exhibit, Texas A\&M University, 2012.

${ }^{32}$ Mughal, M.S., and Ashworth, R., "Uncertainty quantification based receptivity modelling of crossflow instabilities induced by distributed surface roughness in swept wing boundary layers." 43rd AIAA Fluid Dynamics Conference, AIAA, San Diego, CA, 2013.

33 Nitschke-Kowsky, P., "Experimental Studies on the Stability and Transition of Three-dimensional Boundary Layers," National Aeronautics and Space Administration, 1987.

34 van Ingen, J.L., "A suggested semi-empirical method for the calculation of the boundary layer transition region," Delft University of Technology, 1956.

${ }^{35}$ Smith, A.M.O., and Gamberoni, N., "Transition, pressure gradient and stability theory," Douglas Aircraft Company, S-26388, 1956.

${ }^{36}$ BL2D, Software Package, Ver. 3.5, QinetiQ, Unpublished.

${ }^{37}$ CoDS, Software Package, Ver. 5.6, QinetiQ, Unpublished.

38 Hanson, R. E., Buckley, H. P. and P. Lavoie. "Aerodynamic optimization of the flat-plate leading edge for experimental studies of laminar and transitional boundary layers," Experiments of Fluids, Vol. 53, No.4, 2012, pp. 863-871.

${ }^{39}$ Bruun, H.H., "Hot-Wire Anemometry," Oxford University Press, 1995.

${ }^{40}$ Welch, P.D., "The use of Fast Fourier Transform for the estimation of power spectra: A method based on time averaging over short, modified periodograms", IEEE Transactions on Audio and Electroacoustics, Vol. AU-15, No. 2, 1967

${ }^{41}$ Malik, M.R., Li, F. and Chang, C.L., "Crossflow disturbances in three-dimensional boundary layers: nonlinear development, wave interaction and secondary instability," Journal of Fluid Mechanics,Vol.268, 1994, pp.1-36. 